\title{
The Long-Term Effects of Oophorectomy on Cognitive and Motor Aging Are Age Dependent
}

\author{
Walter A. Rocca ${ }^{a, c} \quad$ Brandon R. Grossardt ${ }^{b}$ Demetrius M. Maraganore ${ }^{c}$ \\ Divisions of ${ }^{\mathrm{a}}$ Epidemiology and ${ }^{\mathrm{b}}$ Biostatistics, Department of Health Sciences Research, and ${ }^{\mathrm{c}}$ Department of \\ Neurology, Mayo Clinic College of Medicine, Rochester, Minn., USA
}

\author{
Key Words \\ Oophorectomy · Estrogen · Neuroprotection • Parkinson's \\ disease $\cdot$ Parkinsonism - Dementia $\cdot$ Cognitive impairment • \\ Menopause
}

\begin{abstract}
Background: The evidence for a neuroprotective effect of estrogen in women remains controversial. Objective: We studied the long-term risk of parkinsonism and of cognitive impairment or dementia in women who underwent oophorectomy before menopause. Methods: We conducted a historical cohort study among all women residing in Olmsted County, Minn., USA, who underwent unilateral or bilateral oophorectomy before the onset of menopause for a noncancer indication from 1950 through 1987. Each member of the oophorectomy cohort was matched by age to a referent woman from the same population who had not undergone oophorectomy. In total, we studied 1,252 women with unilateral oophorectomy, 1,075 women with bilateral oophorectomy, and 2,368 referent women. Women were followed for a median of 25-30 years. Parkinsonism was assessed using screening and examination, through a medical recordslinkage system, and through death certificates. Cognitive status was assessed using a structured questionnaire via a direct or proxy telephone interview. Results: The risk of parkinsonism and of cognitive impairment or dementia increased following oophorectomy. In particular, we observed
\end{abstract}

significant linear trends of increasing risk for either outcome with younger age at oophorectomy. Conclusion: Our findings, combined with previous laboratory and epidemiologic findings, suggest that estrogen may have an age-dependent neuroprotective effect. Copyright $\odot 2008$ S. Karger AG, Basel

\section{Introduction}

We previously reported from the Mayo Clinic Cohort Study of Oophorectomy and Aging that bilateral oophorectomy performed before age 45 years is associated with increased overall mortality, as well as mortality caused by neurological and psychiatric disorders [1]. In addition, we reported that women who underwent either unilateral or bilateral oophorectomy have an increased risk of parkinsonism and of cognitive impairment or dementia [2, 3]. In this paper, we provide additional evidence that the effect of oophorectomy on the brain may be age dependent.

\section{Participants and Methods}

Study Sample

The Mayo Clinic Cohort Study of Oophorectomy and Aging included women who underwent unilateral or bilateral oophorectomy and a group of women who did not undergo oophorectomy

\section{KARGER}

๑ 2008 S. Karger AG, Basel

Fax +4161306 1234

E-Mail karger@karger.ch

www.karger.com
Accessible online at:

www.karger.com/ndd
Walter A. Rocca, MD, MPH

Division of Epidemiology, Department of Health Sciences Research

Mayo Clinic, 200 First Street SW

Rochester, MN 55905 (USA)

Tel. +1 507284 3568, Fax +1 507284 1516, E-Mail rocca@mayo.edu 
Fig. 1. Overall design of the Mayo Clinic Cohort Study of Oophorectomy and Aging. The numbers of women included in the follow-up were different for parkinsonism and for cognitive impairment or dementia. The numbers for cognitive impairment or dementia were reduced because only the women who were interviewed were informative. The asterisk indicates that a total of 95 referent women underwent oophorectomy after the index year and from 1950 through 1987. Because these women were included in both cohorts, they were counted only in the oophorectomy cohort regarding vital status at follow-up (2001-2006).

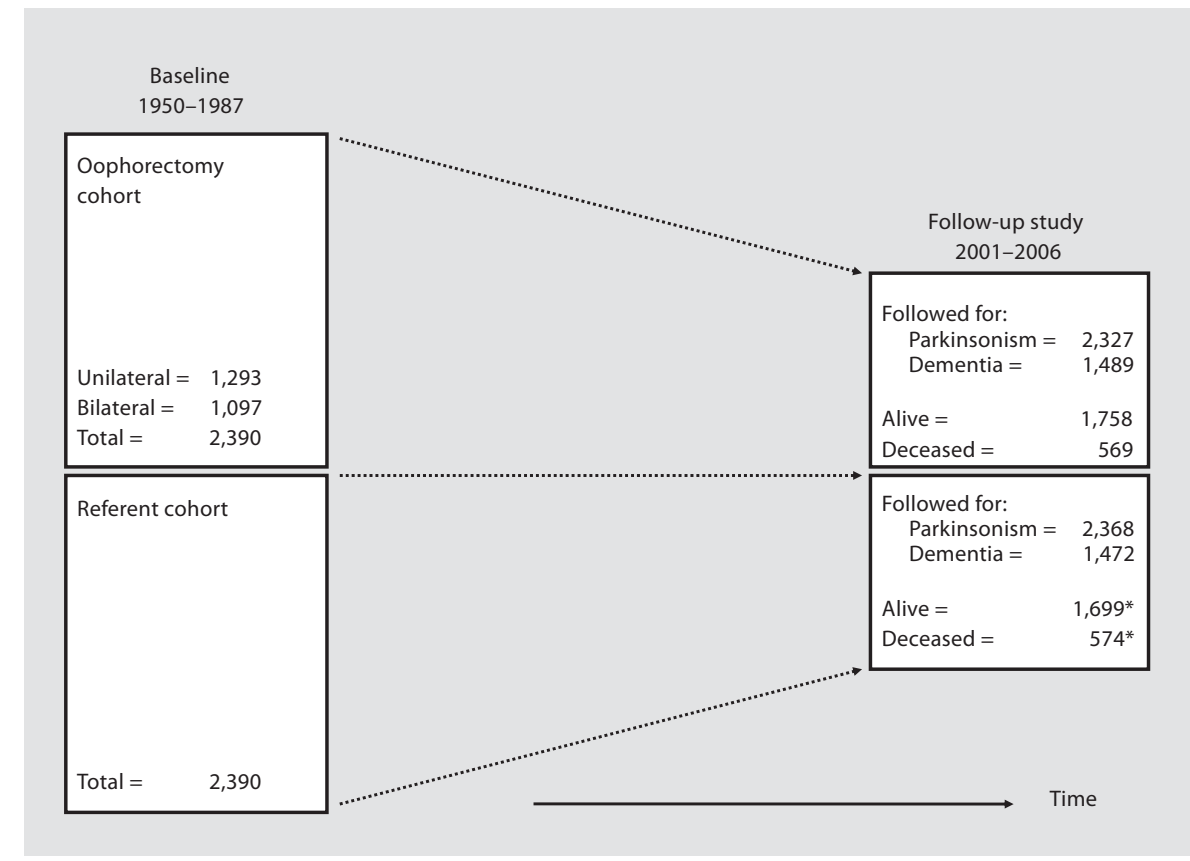

(referent cohort). Both groups were followed using the same methods. All study procedures were approved by the institutional review boards of the Mayo Clinic and of Olmsted Medical Center. All women who were examined as part of the study signed an informed consent form [1].

During the 38-year period from 1950 through 1987, a total of 1,433 women residing in Olmsted County, Minn., USA, underwent a unilateral oophorectomy, and 1,824 underwent a bilateral oophorectomy (including 110 who had a second unilateral oophorectomy). Oophorectomy was defined as complete removal of the ovary. Further details about the oophorectomy cohort were reported elsewhere $[1,4]$. The indication for oophorectomy as defined by the gynecologist at the time of surgery was abstracted from the medical records by trained study nurses. Women from the original cohort were included in the current study if they were born before 1962 (i.e., were at least 40 years old by January 1, 2002), and had their oophorectomy before the onset of menopause (or before age 56 years if age at menopause was unknown). In addition, we excluded women who underwent oophorectomy for ovarian cancer or as treatment for another estrogen-related malignancy (usually breast cancer) because they were at high risk of death shortly after the surgery. Therefore, we considered eligible for the study 1,293 women who underwent unilateral oophorectomy and 1,097 who underwent bilateral oophorectomy (fig. 1).

Potential referent women were identified from a list of residents provided by the records-linkage system of the Rochester Epidemiology Project [5]. This listing has been shown to be complete compared with a random digit dialing telephone sample and with census enumerations [5]. For each woman in the oophorectomy cohort, we defined the year of the surgical procedure as the index year, and we selected (via simple random sampling) one woman from the complete Olmsted County population with the same year of birth, who had survived without oophorectomy to the index year ( $n=2,390$; fig. 1$)$. All women in the population who met these criteria were considered eligible regardless of any possible diseases or risk factors (population-based referent sample).

\section{Follow-Up Procedures}

Women in the oophorectomy and referent cohorts were contacted once during the follow-up study spanning from 2001 through 2006. All women underwent the same procedures to assess the incidence of parkinsonism and of cognitive impairment or dementia following the index year. Parkinsonism was assessed through a two-phase survey with individual telephone screening of the women (or their proxy) for parkinsonism and examination of those who screened positive (when alive), as described in detail elsewhere [2]. In addition, independent of the screening, all women were also followed passively for parkinsonism (but not for cognitive impairment or dementia) through review of medical records in the records-linkage system of the Rochester Epidemiology Project and through death certificates (if deceased) [2]. Cognitive impairment or dementia were assessed using the Telephone Interview for Cognitive Status-modified (12 items; maximum score $=50$ points) or through a brief dementia questionnaire administered to a proxy informant (8 questions), as described in detail elsewhere [3].

All telephone contacts were made by one of 6 specifically trained research assistants and were direct whenever possible; for deceased or otherwise incapacitated women (e.g. deaf, cognitively impaired, or terminally ill), we contacted the best available proxy (most knowledgeable person in the family) [2, 3]. All telephone interviewers and all research team members involved in the ascertainment of the outcome conditions were kept uninformed of the oophorectomy or referent status of women to prevent bias. 


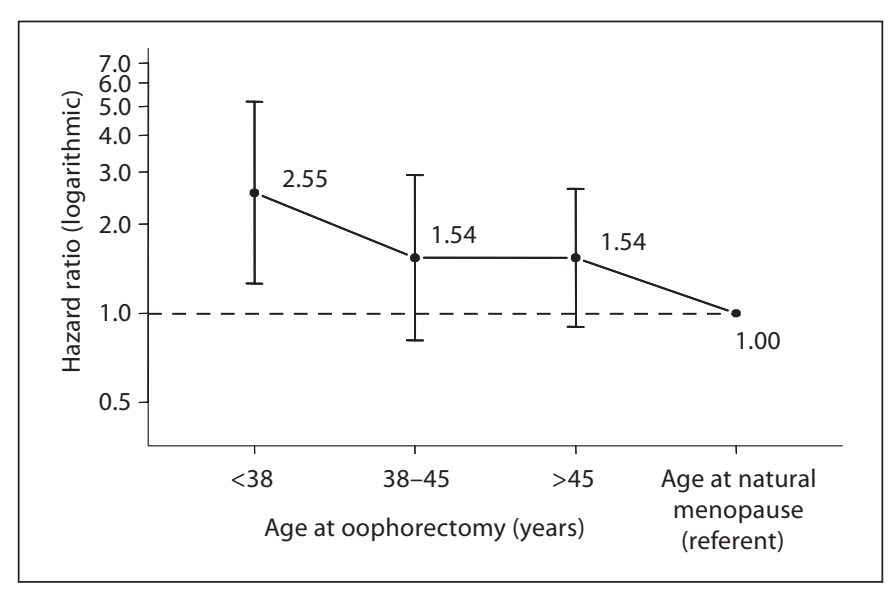

Fig. 2. Risk of parkinsonism by age at oophorectomy (unilateral or bilateral). A test for linear trend in log HRs was significant $(\mathrm{p}=0.01)$. The test for trend included referent women who were assumed to have reached natural menopause around a median age of 50 years $(\mathrm{HR}=1.00)$.

\section{Statistical Analyses}

Women were censored at the end of the study (spanning from 2001 through 2006), at death, or at last contact. We estimated the hazard ratio (HR) using Cox proportional hazards models. The assumption of proportional hazards was assessed by graphical methods and by introducing a time-dependent coefficient in the Cox models [6]. The analyses presented here were stratified by age at surgery (in tertiles). In the analyses for cognitive impairment or dementia, we included only 813 women with unilateral oophorectomy, 676 women with bilateral oophorectomy, and 1,472 referent women because the assessment was limited to telephone interviews (no passive follow-up through medical records or death certificates) [3]. All analyses were conducted using SAS version 8.2 , and statistical tests were performed at the two-tailed alpha level of 0.05 .

\section{Results}

Figure 1 shows the overall design of the study. The number of women included in the follow-up was different for parkinsonism and for cognitive impairment or dementia. The assessment of parkinsonism included both active contacts and passive information. By contrast, the assessment of cognitive impairment or dementia included only direct or proxy interviews. The median follow-up was long (median $=25-30$ years). Figure 2 shows the trend of increasing HRs for parkinsonism in women with younger age at oophorectomy (either unilateral or bilateral). A test for linear trend in the log HRs was significant $(p=0.01)$. Figure 3 shows the trend of increasing HRs for cognitive impairment or dementia in women with young-

Long-Term Effects of Oophorectomy on

Cognitive and Motor Aging

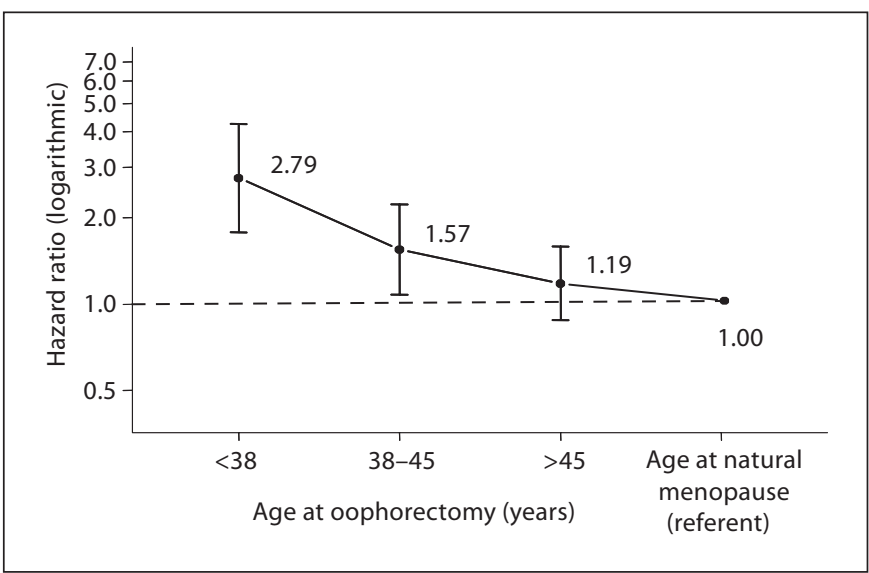

Fig. 3. Risk of cognitive impairment or dementia by age at oophorectomy (unilateral or bilateral). A test for linear trend in the log HRs was significant $(\mathrm{p}<0.0001)$. The test for trend included referent women who were assumed to have reached natural menopause around a median age of 50 years $(\mathrm{HR}=1.00)$.

er age at oophorectomy (either unilateral or bilateral). A test for linear trend in the log HRs was significant $(\mathrm{p}<$ 0.0001).

\section{Discussion and Conclusions}

This study showed an increased long-term risk of parkinsonism and of cognitive impairment or dementia in women who underwent oophorectomy before menopause. The magnitude of the association increased with younger age at oophorectomy. Findings from this study, combined with findings from studies in laboratory animals $[7,8]$ and from other epidemiologic studies [9-11], suggest that the estrogen deficiency caused by bilateral oophorectomy was the initial step in a chain of causality that determined the increased risk of neurological diseases. The increased risk among women who underwent unilateral oophorectomy may be explained by the premature failure of the contralateral ovary caused by the oophorectomy itself or by the concurrent hysterectomy (70.1\% of all unilateral oophorectomies). However, the effect of oophorectomy on the brain may be mediated by other mechanisms such as a deficiency of progesterone or testosterone or by the disruption of the hypothalamic-pituitary-gonadal axis. Finally, one or several susceptibility genes may be involved in causing the association. Further discussion of these biological mechanisms was provided elsewhere $[2,3]$. 
Our findings for parkinsonism and for cognitive impairment or dementia in aggregate suggest that the putative neuroprotective effect of estrogen may be general and may involve multiple mechanisms and multiple neuronal populations. In addition, our findings suggest that the putative neuroprotective effect of estrogen may be age dependent and that there may be a critical age window for neuroprotection. The concept of a critical age window for neuroprotection has been proposed by several other investigators [7-11].

\section{Acknowledgments}

This research was supported by the National Institute of Neurological Disorders and Stroke (grant R01 NS33978) and the National Institute of Arthritis and Musculoskeletal and Skin Diseases (grant R01 AR30582).

\section{References}

$>1$ Rocca WA, Grossardt BR, de Andrade M, Malkasian GD, Melton LJ 3rd: Survival patterns after oophorectomy in premenopausal women: a population-based cohort study. Lancet Oncol 2006;7:821-828.

2 Rocca WA, Bower JH, Maraganore DM, Ahlskog JE, Grossardt BR, de Andrade M, Melton LJ 3rd: Increased risk of parkinsonism in women who underwent oophorectomy before menopause. Neurology 2007, Epub ahead of print.

3 Rocca WA, Bower JH, Maraganore DM, Ahlskog JE, Grossardt BR, de Andrade M, Melton LJ 3rd: Increased risk of cognitive impairment or dementia in women who underwent oophorectomy before menopause. Neurology 2007;69:1074-1083.
4 Melton LJ 3rd, Bergstralh EJ, Malkasian GD, O’Fallon WM: Bilateral oophorectomy trends in Olmsted County, Minnesota, 1950-1987. Epidemiology 1991;2:149-152.

$\checkmark 5$ Melton LJ 3rd: History of the Rochester Epidemiology Project. Mayo Clin Proc 1996;71: 266-274.

6 Therneau TM, Grambsch PM: Modeling Survival Data: Extending the Cox Model. New York, Springer, 2000.

7 Gibbs RG: Long-term treatment with estrogen and progesterone enhances acquisition of a spatial memory task by ovariectomized aged rats. Neurobiol Aging 2000;21:107116.
8 Morrison JH, Brinton RD, Schmidt PJ, Gore AC: Estrogen, menopause, and the aging brain: how basic neuroscience can inform hormone therapy in women. J Neurosci 2006;26:10332-10348.

$\checkmark 9$ Zandi PP, Carlson MC, Plassman BL, WelshBohmer KA, Mayer LS, Steffens DC, Breitner JC: Hormone replacement therapy and incidence of Alzheimer disease in older women: the Cache County Study. JAMA 2002;288: 2123-2129.

10 Henderson VW, Benke KS, Green RC, Cupples LA, Farrer LA: Postmenopausal hormone therapy and Alzheimer's disease risk: interaction with age. J Neurol Neurosurg Psychiatry 2005;76:103-105.

11 Siegfried T: Neuroscience: it's all in the timing. Nature 2007;445:359-361. 